\title{
Multiproduct Pricing Made Simple
}

\author{
Mark Armstrong \\ All Souls College and University of Oxford
}

John Vickers

All Souls College and University of Oxford

\begin{abstract}
We study multiproduct firms in the contexts of unregulated monopoly, regulated monopoly, and Cournot oligopoly. Using the concept of consumer surplus as a function of quantities (rather than prices), we present simple formulas for optimal prices and show that Cournot equilibrium exists and corresponds to a Ramsey optimum. We then discuss a tractable class of preferences that involve a generalized form of homotheticity. Profit-maximizing quantities are proportional to efficient quantities. We discuss optimal monopoly regulation when the firm has private information about its cost vector and find situations in which optimal regulation leaves relative price decisions to the firm.
\end{abstract}

\section{Introduction}

The theory of multiproduct pricing is a large and diverse subject. Unlike the single-product case, a multiproduct firm must decide about the structure of its relative prices as well as its overall price level. Classical questions include the characterization of optimal pricing by a multiproduct monopolist seeking to maximize profit—or, as with Ramsey pricing, the most efficient way to generate a specified level of profit - when its choice for one price must take into account its impact on demand for other products. Additional complexities arise in oligopoly when a multiproduct firm

We are grateful to Paolo Bertoletti, Jim Mirrlees, Stephen Morris, Jonas Müller-Gastell, Robert Ritz, José Scheinkman, Konrad Stahl, Tommaso Valetti, Glen Weyl, and four referees for helpful comments.

Electronically published July 2, 2018

[ Journal of Political Economy, 2018, vol. 126, no. 4]

(C) 2018 by The University of Chicago. All rights reserved. 0022-3808/2018/12604-0010\$10.00 
needs to choose prices or quantities to reflect both intrafirm substitution (or complementarity) features and interfirm interactions. Optimal regulation of a multiproduct firm with private information about its costs, say, must take into account not just its likely average cost across all products but its pattern of relative costs.

In this paper we show how these issues can be illuminated by studying consumer preferences in terms of consumer surplus considered as a function of quantities (rather than the more familiar function of prices). ${ }^{1}$ In Section II we show how profit-maximizing and other Ramsey prices, as well as prices in symmetric Cournot equilibrium, can be expressed as a markup over marginal costs proportional to the gradient of this surplus function. In particular, a product's price is below marginal cost when consumer surplus decreases with the supply of this product. We also show how a Cournot equilibrium corresponds to an appropriate Ramsey optimum, and vice versa, which enables us to construct and demonstrate existence of Cournot equilibrium in many cases.

A well-known feature of Ramsey pricing is that when required departures of optimal quantities from efficient quantities are small, then optimal quantities are approximately proportional to the efficient quantities. Thus, a reasonable rule of thumb is often to scale down quantities equiproportionately relative to efficient quantities rather than to increase prices equiproportionately above marginal cost. For larger departures of prices from costs, though, optimal quantities are generally not proportional to efficient quantities. In Section III, we specialize the demand system so that consumer surplus is a homothetic function of quantities, which implies that relative quantities (or relative price-cost markups) do not depend on the weight placed on profit in the Ramsey objective. As shown in Section III.B, this is quite a flexible class of demand systems (and much broader than the class in which consumer surplus is homothetic in prices), and as well as the obvious case of gross utility being homothetic in quantities, it includes linear and logit demands.

In Section III.C, we show that this homotheticity property, together with assuming constant returns to scale, simplifies the analysis by allowing a multiproduct problem to be decomposed into two steps: first calculate the efficient quantities that correspond to marginal cost pricing and, second, solve for the scale factor by which to reduce the efficient quantities. There is a much richer pattern of price-cost markups when consumer surplus is homothetic than with homothetic gross utility (when markups are equiproportionate), but the analysis is readily tractable. We show how monopoly (and symmetric Cournot oligopoly) prices vary with cost parameters. In some leading examples there is zero "cross-

\footnotetext{
${ }^{1}$ Regarding consumer surplus as a function of quantities is apparently uncommon in the literature. However, in the single-product context Bulow and Klemperer (2012) show this to be a valuable perspective.
} 
cost" pass-through - so the most profitable price of each product depends only on its own cost - and more generally there are simple formulas for the size and sign of cross-cost effects.

The fact that a profit-maximizing firm has efficient incentives with respect to relative quantities, though of course not the average quantity, has implications, moreover, for regulation of multiproduct monopoly (Sec. IV). It suggests that, in our class of demand systems, it might sometimes be optimal for regulation to allow the monopolist considerable discretion over the pattern of relative quantities (or prices). If the probability distribution over costs is such that relative costs and average costs are stochastically independent, this intuition is precisely correct, and it is optimal for the choice of relative prices to be delegated to the firm.

Related literature.-Baumol and Bradford (1970), and the many references therein, discuss the economic principles of multiproduct pricing based on Ramsey's (1927) classic analysis of commodity taxation. They suggest that it is plausible that "the damage to welfare resulting from departures from marginal cost pricing will be minimized if the relative quantities of the various products sold are kept unchanged from their marginal cost pricing proportions" (271). Ramsey had observed that with linear demands this principle holds not only for small departures from marginal cost pricing, and one aim of our paper is to make this intuition precise in a broader class of demand systems.

Cournot oligopoly is studied in a rich literature on single-product firms; see Vives (1999, chap. 4) for an overview of existence, uniqueness, and comparative statics of Cournot equilibria. Sometimes a Cournot oligopoly operates as if it collectively maximizes an objective. Bergstrom and Varian (1985) observe that a symmetric oligopoly operates to maximize a weighted sum of profit and consumer surplus, while Slade (1994) and Monderer and Shapley (1996) note that oligopolists sometimes maximize a more abstract "potential function." This is useful as it converts the fixed-point problem of calculating equilibrium quantities into a simpler optimization problem. In proposition 1 we extend this analysis to cover multiproduct cases and, like Bergstrom and Varian (1985), show that oligopolists' equilibrium behavior corresponds to maximization of an appropriate Ramsey objective. However, the Ramsey objective is not a potential function for the Cournot game, and neither is there best-response equivalence (Morris and Ui 2004).

The literature on multiproduct oligopoly is more extensive for Bertrand competition than for Cournot competition. ${ }^{2}$ Anderson and de

${ }^{2}$ Johnson and Myatt (2006) study multiproduct Cournot oligopoly in the context of quality upgrades. In leading cases each upgrade market can be treated independently, which greatly simplifies the analysis. De Fraja (1996, sec. 3) shows that if equilibrium exists in his multiproduct Cournot duopoly model, it is symmetric. In particular, firms supply identical product lines, though some product varieties might not be supplied. 
Palma (1992) prove the existence of symmetric Bertrand equilibrium with multiproduct firms using the nested logit model of demand. A focus of their analysis, which (unlike ours below) has fixed costs per product, is the range of products supplied. Nocke and Schutz (2018) take an aggregative games approach to analyze Bertrand competition in a discrete/continuous choice model in which consumer surplus is an additively separable function of prices (as with logit). A connection between Bertrand equilibrium and Ramsey pricing is shown by Bliss (1988) and Armstrong and Vickers (2001, sec. 2) in a model in which consumers buy all products from one firm or another, so there is one-stop shopping. Firms therefore compete, by way of linear prices, in terms of the surplus they offer their customers. If consumers have the same gross utility function but differ in their brand preferences for the various firms, each firm's strategy can be broken down into two steps: (i) the Ramsey problem of choosing the most profitable way to deliver a given surplus to a customer and (ii) how much surplus to offer. Even with market power, a firm will therefore choose efficient relative prices at step i.

Weyl and Fabinger (2013) discuss the pass-through of costs to prices and its various applications in settings of monopoly and imperfect competition with single-product firms. Within the marketing literature on retailing, a major theme is the extent to which wholesale cost shocks (such as temporary promotions) are passed through into retail prices. Besanko, Dubé, and Gupta (2005) empirically examine the patterns of cost passthrough in a large supermarket chain. They find that own-cost proportional pass-through is more than 60 percent for most product categories (and sometimes more than 100 percent), while cross-cost pass-through can take either sign. Amir et al. (2016) show that a monopolist facing a linear demand system chooses prices such that cross-cost pass-through is precisely zero. Moorthy (2005) analyzes a theoretical model in which two retailers compete to supply two products to consumers, and as well as cost pass-through within a retailer, he discusses how cost shocks to the rival affect a firm's prices. The sign of most of the pass-through effects depends in an opaque way on the features of various matrices. In Section III.C, our demand system yields some relatively simple multiproduct pass-through relationships.

The optimal regulation of multiproduct monopoly is analyzed by Laffont and Tirole (1993, chap. 3). In their main model the regulator cannot observe cost-reducing effort or the firm's underlying cost type, but cost outturns are observable. The analysis of regulation in Section IV below does not consider effort incentives, but is for the situation studied by Baron and Myerson (1982) in which the regulator cannot observe the firm's exogenous costs. We extend this model to cover multiproduct situations in which a vector of marginal costs is unobserved by the regulator. Building on the approach in Armstrong (1996) and Armstrong and Vick- 
ers (2001), we describe a class of situations in which it is optimal to control only the firm's average price, leaving it free to choose relative prices to reflect relative costs.

\section{A General Analysis of Multiproduct Pricing}

A. The Framework

Suppose there are $n \geq 2$ products, where the quantity of product $i$ is denoted $x_{i}$ and the vector of quantities is $x=\left(x_{1}, \ldots, x_{n}\right)$. Consumers have quasi-linear utility, which implies that demand can be considered to be generated by a single representative consumer with gross utility function $u(x)$ defined on a (full-dimensional) convex region $R \subset \mathbb{R}_{+}^{n}$, which includes zero, where $u(0)=0$ and $u$ is increasing and strictly concave. (We might have $R=\mathbb{R}_{+}^{n}$, so that utility is defined for all nonnegative quantity bundles.) We suppose that $u$ is twice continuously differentiable in the interior of $R$, although marginal utility might be unbounded as some quantities tend to zero.

These products are sold to the consumer via linear prices, and faced with the price vector $p=\left(p_{1}, \ldots, p_{n}\right)$, the consumer chooses quantities $x \in R$ to maximize $u(x)-p \cdot x$. (Here, $a \cdot b \equiv \sum_{i=1}^{n} a_{i} b_{i}$ denotes the dot product of two vectors $a$ and $b$.) The price vector that induces interior quantity vector $x \in R$ to be demanded, that is, the inverse demand function $p(x)$, is

$$
p(x) \equiv \nabla u(x)
$$

where we use the gradient notation $\nabla f(x) \equiv\left(\partial f(x) / \partial x_{1}, \ldots, \partial f(x) / \partial x_{n}\right)$ for the vector of partial derivatives of a function $f$. To ensure we can invert $p(x)$ to obtain the demand function $x(p)$, we assume that the matrix of second derivatives of $u$, which we write as $D p(x)$, is nonsingular (and hence negative definite). The revenue generated from quantity vector $x$ is

$$
r(x)=x \cdot \nabla u(x),
$$

while the surplus retained by the consumer from $x$ is

$$
s(x) \equiv u(x)-r(x) .
$$

One of this paper's aims is to show the usefulness of the function $s(x)$ for analyzing multiproduct pricing.

The derivative of $s$ can be expressed as

$$
\nabla s(x)=\left.\frac{d}{d k} p(x / k)\right|_{k=1},
$$


so that an equiproportionate contraction in quantities $x$ moves the price vector in the direction $\nabla s(x)$, that is, normal to the surface $s(x)=$ constant. To see (2), note that

$$
\begin{aligned}
\frac{\partial}{\partial x_{i}} s(x) & =\frac{\partial}{\partial x_{i}}[u(x)-r(x)]=-\sum_{j} x_{j} \frac{\partial}{\partial x_{i}} p_{j}(x)=-\sum_{j} x_{j} \frac{\partial}{\partial x_{j}} p_{i}(x) \\
& =\left.\frac{d}{d k} p_{i}(x / k)\right|_{k=1},
\end{aligned}
$$

where the third equality follows from the symmetry of cross-derivatives of $p(x)$. Unlike consumer surplus expressed as a function of prices-which is necessarily a decreasing function of prices-here $s(x)$ can increase or decrease with $x_{i}{ }^{3}$ From (2), a sufficient condition for $s$ to increase with $x_{i}$ is that $p_{i}$ decrease with all $x_{j}$, which is the case if products are gross substitutes (see Vives 1999, sec. 6.1).

\section{B. The Ramsey Monopoly Problem}

Now suppose that these products are supplied by a monopolist with differentiable cost function $c(x)$. To sidestep issues of fixed costs and the potential undesirability of producing at all, both with monopoly and in the later analysis of Cournot oligopoly, we suppose that

$$
c(0)=0 \text { and } c(x) \text { is convex. }
$$

Consider the Ramsey problem of choosing quantities to maximize a weighted sum of profit and consumer surplus. If $\alpha \leq 1$ is the relative weight on consumer surplus, the Ramsey objective is

$$
[r(x)-c(x)]+\alpha s(x)=u(x)-c(x)-(1-\alpha) s(x) .
$$

This includes as polar cases profit maximization $(\alpha=0)$ and total surplus maximization $(\alpha=1)$. Standard comparative statics shows that optimal consumer surplus, $s(x)$, in this Ramsey problem weakly increases with $\alpha$, while optimal profit, $r(x)-c(x)$, weakly decreases with $\alpha$. Total surplus $u(x)-c(x)$ is a strictly concave function that is maximized at quantities $x^{w}$ that involve prices equal to marginal costs, so that $p\left(x^{w}\right)=$ $\nabla c\left(x^{w}\right)$. (We assume the efficient quantities are finite. Assumption [3] ensures that the firm at least breaks even with marginal-cost pricing.) More

${ }^{3}$ However, the above expressions imply that $s(x)$ must increase as all quantities are increased equiproportionately. (In particular, when $n=1$, surplus $s(x)$ must increase with scalar quantity $x$.) In addition, while consumer surplus is necessarily convex as a function of prices, it is ambiguous whether $s(x)$ is convex or concave (or neither) as a function of quantities. 
generally, assuming positive supply of all products, ${ }^{4}$ the Ramsey problem with weight $\alpha$ has a first-order condition for optimal quantities given by

$$
p(x)=\nabla c(x)+(1-\alpha) \nabla s(x) .
$$

Expression (5) is an alternative, and arguably more transparent, formulation of the insight in Baumol and Bradford (1970, sec. 8) that the gap between price and marginal cost should be proportional to the gap between marginal revenue and marginal cost. It shows that when $\alpha<1$, the optimal departure of price from marginal cost is proportional to $\nabla s(x)$. In particular, the Ramsey price for product $i$ is above its marginal cost if surplus $s(x)$ increases with $x_{i}$ at optimal quantities, while using the product as a "loss leader" is optimal if $s(x)$ decreases with $x_{i}$. As we will see in Section III.B, there are also cases in which $s$ depends only on the quantities of a subset of products, in which case (5) indicates that the remaining products should be priced at marginal cost. Thus, the function $s$ succinctly determines when it is optimal to set a price above, below, or equal to marginal cost.

Further insights can be obtained when the cost function $c(x)$ is homogeneous degree 1 . In this case, when $\alpha<1$, expression (5) implies that at a Ramsey optimum a small equiproportionate reduction in quantities will induce an equiproportionate increase in price-cost markups. To see this, observe that at quantities $x$ that satisfy (5), we have

$$
\left.\frac{d}{d k}[p(x / k)-\nabla c(x / k)]\right|_{k=1}=\nabla s(x)=\frac{1}{1-\alpha} \times[p(x)-\nabla c(x)] .
$$

Here, the first equality uses $(2)$ and that $\nabla c(x)$ is homogeneous degree 0 , while the second follows from (5). In words, a 1 percent reduction in Ramsey quantities amplifies each price-cost markup by $1 /(1-\alpha)$ percent. $^{5}$

A second insight with constant returns to scale is that choosing $x=$ $\alpha x^{w}$ approximately solves the Ramsey problem (4) when $\alpha$ is close to one. Expression (2) implies that $p(\alpha x)-p(x) \approx(1-\alpha) \nabla s(x)$ when $\alpha \approx$ 1 , in which case

$$
\begin{aligned}
p\left(\alpha x^{w}\right) & \approx p\left(x^{w}\right)+(1-\alpha) \nabla s\left(x^{w}\right) \\
& =\nabla c\left(x^{w}\right)+(1-\alpha) \nabla s\left(x^{w}\right) \\
& =\nabla c\left(\alpha x^{w}\right)+(1-\alpha) \nabla s\left(x^{w}\right) \\
& \approx \nabla c\left(\alpha x^{w}\right)+(1-\alpha) \nabla s\left(\alpha x^{w}\right),
\end{aligned}
$$

\footnotetext{
${ }^{4}$ If some products are not optimally supplied, then (5) holds for products in positive supply.

${ }^{5}$ In the context of commodity taxation, Mirrlees (1976, sec. 2) shows that at a Ramsey optimum a small equiproportionate increase in price-cost margins will cause quantities to fall equiproportionately.
} 
so that $x=\alpha x^{w}$ approximately satisfies condition (5). Here, the first strict equality follows from the efficiency of quantities $x^{w}$, the second follows from the homogeneity of $c(\cdot)$, while the final approximation follows by taking a Taylor expansion of the function $(1-\alpha) \nabla s\left(\alpha x^{w}\right)$ about $\alpha=1$. In sum, in the Ramsey problem with constant returns to scale and $\alpha \approx$ 1 , the efficient quantities should be scaled back equiproportionately with the factor $\alpha$. This accords with the Ramsey tax insight that equiproportionate quantity reductions are optimal when revenue requirements are small.

Without making further assumptions, there is little reason to expect that this result for $\alpha \approx 1$ extends to the situation in which a monopolist maximizes profit $(\alpha=0)$, and in general profit-maximizing quantities are not proportional to efficient quantities. (Indeed, it is perfectly possible for the monopolist to supply more of one product compared with efficient supply.) If the demand system was such that Ramsey quantities for different $\alpha$ all lay on the same ray from the origin, and if the cost function had constant returns to scale, then expression (6) implies that relative price-cost margins do not vary with $\alpha$. In turn, (5) then implies that $\nabla s(x)$ does not change direction as $x$ is scaled up or down equiproportionately, that is, that $s(x)$ is a homothetic function of $x$. In Section III, we focus on demand systems with precisely that feature.

\section{Cournot Oligopoly}

A natural development of our framework is to the Cournot oligopoly setting with $m$ symmetric firms that each supply the full range of products and have the same cost function $c(\cdot)$ satisfying (3). Our main result in this context is that equilibrium in this $m$-player game is closely related to an appropriate Ramsey optimum. Assumption (3) implies that a least-cost way for the industry to supply total quantity vector $x$ is to split this quantity equally between the $m$ firms so that total cost is $m c((1 / m) x)$. In this case the Ramsey objective (4) becomes

$$
u(x)-m c\left(\frac{1}{m} x\right)-(1-\alpha) s(x),
$$

so that the corresponding first-order condition for the optimal vector of total quantity $x$ is

$$
p(x)=\nabla c\left(\frac{1}{m} x\right)+(1-\alpha) \nabla s(x) .
$$

Consider a candidate symmetric Cournot equilibrium in which each firm supplies quantity vector $(1 / m) x$ (so that $x$ is total supply). Then a firm must maximize its own profit 


$$
x^{j} \cdot p\left(\frac{m-1}{m} x+x^{j}\right)-c\left(x^{j}\right)
$$

by choosing $x^{j}=(1 / m) x$, which from expression (2) has the first-order condition

$$
p(x)=\nabla c\left(\frac{1}{m} x\right)+\frac{1}{m} \nabla s(x) .
$$

Comparing (9) with (8) reveals that a symmetric Cournot equilibrium, if it exists, has the same first-order conditions as the Ramsey problem (7) when the weight on consumers is $\alpha=(m-1) / m$.

Putting this a different way, the gradient of industry profit $x \cdot p(x)-$ $m c((1 / m) x)$ with respect to changes in total supply $x$ is $p(x)-$ $\nabla c((1 / m) x)+x \cdot D p(x)$, while the first-order condition for $x$ to be total supply in a symmetric Cournot equilibrium is

$$
p(x)-\nabla c\left(\frac{1}{m} x\right)+\frac{1}{m} x \cdot D p(x)=0 .
$$

Therefore, the first-order condition for $x$ to be supplied in symmetric Cournot equilibrium is that the gradient of industry profit at $x$ be $[(m-$ $1) / m] x \cdot D p(x)$. In turn, expression (2) implies that this gradient be $[(m-1) / m] \nabla s(x)$, which corresponds to the first-order condition for $x$ to maximize the Ramsey objective with weight on consumers given by $\alpha=(m-1) / m$.

Moving beyond these first-order arguments, the following result establishes the existence and symmetry of Cournot equilibrium. (The proof can be found in the appendix.)

Proposition 1. Suppose there are $m$ Cournot competitors, each of which has the same cost function $c(x)$ satisfying (3). Then there exists a symmetric Cournot equilibrium in which quantities maximize the Ramsey objective (7) with $\alpha=(m-1) / m$. There are no asymmetric equilibria. If in addition $r(x)$ is concave, there is only one equilibrium.

Thus, with symmetric convex cost functions, the Ramsey optimum with $\alpha=(m-1) / m$ corresponds to a symmetric Cournot equilibrium, and there are no asymmetric equilibria. In this sense, the Cournot problem and the Ramsey problem are the same. This generalizes to the multiproduct context the second remark in Bergstrom and Varian (1985): that equilibrium total quantity in the symmetric single-product Cournot model can be considered to maximize a weighted average of profit and consumer surplus. ${ }^{6}$ It is not the case, however, that a Cournot firm's profit

\footnotetext{
${ }^{6}$ Bergstrom and Varian (1985) relax the Cournot assumption to allow for a "conjectural variation" term whereby each firm conjectures that total quantity increases by $1+\gamma$ units if its output increases by one unit. The resulting "equilibrium" corresponds to maximizing the Ramsey objective with $\alpha=(m-1-\gamma) / m$.
} 
equals the Ramsey objective plus a function of other firms' actions. In general, the Cournot model is not a potential game. ${ }^{7}$ Neither does the more general property of best-response equivalence-in the sense of Morris and Ui (2004) — hold as between the Cournot and Ramsey objectives. There is a sense in which the Cournot firm likes to be "closer" to other firms' choices than the Ramsey objective. This relative preference for symmetry, as expressed by (A1) in the appendix, however, ensures that Ramsey quantities are the Cournot best response to Ramsey quantities.

When $c(x)$ is homogeneous degree 1 , the number of suppliers has no impact on industry costs and the Ramsey objectives (4) and (7) coincide. In this case, since consumer surplus in the Ramsey problem (4) increases, and profit decreases, with $\alpha$, we deduce that as the number of competitors increases, a symmetric Cournot equilibrium delivers more surplus to consumers and involves lower industry profit, and with many firms the equilibrium quantities are approximately $[(m-1) / m] x^{w}$ (where $x^{w}$ is the efficient quantity vector). One can also study how equilibrium prices depend on marginal costs by studying the simpler Ramsey problem, as we do below in Section III.C.

The analysis in proposition 1 assumes firms are symmetric. Among other issues, this assumption means one cannot study the impact of firm-specific cost shocks, for instance, but only symmetric industrywide cost shocks. When Cournot equilibria exist in asymmetric settings, however, it is straightforward to obtain first-order conditions for equilibrium prices. For example, suppose that each firm has constant marginal costs, and firm $j$ has the marginal cost vector $c^{j}=\left(c_{1}^{j}, \ldots, c_{n}^{j}\right)$. Then if all firms supply all products in equilibrium, an argument similar to (9) shows that equilibrium prices satisfy

$$
p(x)=\frac{1}{m} \sum_{j} c^{j}+\frac{1}{m} \nabla s(x),
$$

where $(1 / m) \sum_{j} c^{j}$ is the industry average vector of marginal costs. ${ }^{8}$ Thus, the Cournot equilibrium here corresponds to a the Ramsey optimum with weight on consumers $\alpha=(m-1) / m$ and a hypothetical monopolist with cost function $c(x)=(1 / m)\left(\sum_{j} c^{j}\right) \cdot x$.

The general analysis in this section has introduced the consumer surplus function $s(x)$ and shown its usefulness in analyzing the Ramsey mo-

\footnotetext{
However, Slade (1994) and Monderer and Shapley (1996) showed that there exists a potential function for the single-product Cournot model with linear demand.

${ }^{8}$ This generalizes the first remark in Bergstrom and Varian (1985) - that equilibrium industry output depends only on the average marginal cost in the industry, not its distribution-to multiple products. One can show (i) that this Cournot equilibrium exists if inverse demands $p_{i}(x)$ are each weakly concave and (ii) that the cost vectors $c^{j}$ are "close enough" so that each firm supplies all products in equilibrium.
} 
nopoly problem and, by extension, the symmetric Cournot oligopoly problem. In the rest of the paper we develop the analysis by supposing that $s$ is homothetic in $x$, in which case the feature of equiproportionate quantity reduction that appeared locally (for $\alpha \approx 1$ in the Ramsey problem or large $m$ in the Cournot equilibrium) in the analysis above holds globally.

\section{A Family of Demand Systems}

\section{A. Homothetic Consumer Surplus}

The family of demand systems on which we now focus is characterized by the property that consumer surplus $s(x)$ is a homothetic function of quantities $x$. We first describe which demand systems have this property:

Proposition 2. Consumer surplus $s(x)$ is homothetic in $x$ if and only if utility $u(x)$ can be written in the form

$$
u(x)=h(x)+g(q(x)),
$$

where $h(\cdot)$ and $q(\cdot)$ are homogeneous degree 1 functions and $g(\cdot)$ is concave with $g(0)=0$.

For the remainder of the paper we assume that utility $u(x)$, as well as being concave, can be written in the form (11). Note we must have $g(0)=0$ and $g$ concave in $q$ given that $u(0)=0$ and $u(\cdot)$ is concave in $x$. Differentiating expression (11) implies that in our family inverse demand takes the form

$$
p(x)=\nabla h(x)+g^{\prime}(q(x)) \nabla q(x) .
$$

Therefore, since $\nabla h$ and $\nabla q$ are homogeneous degree 0 , an equiproportionate change in quantities moves the price vector along a straight line in the direction $\nabla q(x)$. In geometric terms, quantity vectors on the ray joining $x$ to the origin correspond to price vectors that lie on the straight line starting at $p(x)$ pointing in the direction $\nabla q(x)$.

It follows that revenue $r(x)=x \cdot p(x)$ is

$$
r(x)=h(x)+g^{\prime}(q(x)) q(x),
$$

where we used the fact that $x \cdot \nabla h(x) \equiv h(x)$ for a homogeneous degree 1 function. This is a similar form to (11), and because of this, a multiproduct monopolist's problem of maximizing profit is closely connected to the consumer's problem of maximizing surplus, where prices in the consumer's problem correspond to marginal costs in the firm's problem.

Consumer surplus $s(x)=u(x)-r(x)$ is then

$$
s(x)=g(q(x))-g^{\prime}(q(x)) q(x),
$$


which is homothetic since $s(x)$ is an increasing function of the homogeneous function $q(x) .{ }^{9}$ (Since $g$ is concave, $g(q)-g^{\prime}(q) q$ is an increasing function.) This completes the proof that utility of the form (11) has consumer surplus homothetic in quantities. The "only if" part of proposition 2 is proved in the appendix.

The set of demand systems in which consumer surplus is homothetic in quantities is much broader than that in which consumer surplus is homothetic in prices. Expressed as a function of prices, consumer surplus is the convex function $v(p)=\max _{x \geq 0}\{u(x)-p \cdot x\}$. Duality implies that $u(x)$ can be recovered from $v(p)$ using the procedure $u(x)=$ $\min _{p \geq 0}\{v(p)+p \cdot x\}$, and if $v(p)$ is homothetic in $p$, then $u(x)=$ $\min _{p \geq 0}\{v(p)+p \cdot x\}$ is homothetic in $x$. Thus, the utility functions such that consumer surplus is homothetic in prices are simply the homothetic utility functions, that is, where $h \equiv 0$ in (11), which is a subset of the family of preferences we study. In Section III.B, we discuss familiar instances of the family (11) that do not have homothetic utility.

There are three degrees of freedom when choosing a demand system within the class - $q(x), h(x)$, and $g(q)$-and expression (11) provides a useful tool kit for constructing tractable multiproduct demand systems with particular desired properties. For this purpose it is useful to know conditions for the resulting utility function $u$ to be concave. Sufficient conditions to ensure that $u$ in (11) is concave are that $h$ and $g$ are concave and either (i) $q$ is concave and $g$ is increasing, (ii) $q$ is convex and $g$ is decreasing, or (iii) $q$ is linear in $x$ (which allows $g$ to be nonmonotonic).$^{10}$

We now discuss in more detail the implications of the utility specification (11) for the corresponding demand system. Given prices $p$, the representative consumer with utility (11) can maximize her surplus with a simple two-step procedure. We can write the quantity vector $x$ in the form

$$
x \equiv q(x) \times \frac{x}{q(x)} .
$$

Here, $x / q(x)$ is homogeneous degree 0 and depends only on the ray from the origin on which $x$ lies (i.e., it is the direction of the quantity vector), while $q(x)$ is homogeneous degree 1 and measures how far along that ray $x$ lies, and so the decomposition (15) represents a form of "polar

\footnotetext{
${ }^{9}$ It follows that surplus $s$ increases with $x_{i}$-and the Ramsey price for product $i$ is above marginal cost in (5) -if and only if $q(\cdot)$ increases with $x_{i}$.

${ }^{10}$ If $q(x)$ and $h(x)$ are both linear, (12) implies that prices are a function of $q$. Cournot oligopoly is then an aggregative game in the sense that each player's payoff depends on its own action (i.e., its quantity vector) and the composite quantity $q$. Nocke and Schutz (2018) take an aggregative games approach to multiproduct oligopoly with Bertrand competition.
} 
coordinates" for the quantity vector $x$. (The coordinate $x / q(x)$ lies on the $(n-1)$-dimensional surface $q \equiv 1$.) Henceforth we refer to $q(x)$ as "composite" quantity and $x / q(x)$ as the vector of "relative" quantities.

The consumer's surplus with arbitrary quantities $x$ and prices $p$ can be written in terms of the coordinates (15) as

$$
h(x)+g(q(x))-p \cdot x=g(q(x))-q(x) \frac{p \cdot x-h(x)}{q(x)} .
$$

Since the function $p \cdot x-h(x)$ is homogeneous degree $1,[p \cdot x-h(x)] / q(x)$ depends only on the relative quantities $x / q(x)$. Since consumer surplus in (16) is decreasing in $[p \cdot x-h(x)] / q(x)$, the consumer should choose relative quantities to minimize this term, regardless of her choice for composite quantity. Therefore, write

$$
\phi(p) \equiv \min _{x \geq 0}: \frac{p \cdot x-h(x)}{q(x)},
$$

which is an increasing and concave function of $p$. The envelope theorem implies that its derivative is the optimal choice of relative quantities given $p$, so if we write $x^{*}(p) \equiv \nabla \phi(p)$, the consumer facing prices $p$ chooses relative quantities $x^{*}(p) .{ }^{11}$

Given the relative quantities, $x^{*}(p)$, the optimal choice of composite quantity, say $Q$, is easily derived. Consumer surplus in (16) with the optimal relative quantities is $g(Q)-Q \phi(p)$, which is a concave function of $Q$. Write $\hat{Q}(\phi)$ for the composite quantity that maximizes $g(Q)-Q \phi$, which is necessarily weakly downward sloping, so that $\hat{Q}(\phi(p))$ is the demand for composite quantity given the price vector $p$. Price vectors with the same $\phi(p)$ induce the consumer to choose the same composite quantity $Q$, and so $\phi(p)$ is the "composite" price that corresponds to composite quantity $q(x)$. Since the consumer chooses relative quantities $x^{*}(p)$ and composite quantity $\hat{Q}(\phi(p))$, from (15) the vector of quantities demanded at prices $p$ - that is, her demand function $x(p)$-is

$$
x(p)=\hat{Q}(\phi(p)) \times x^{*}(p) .
$$

Here, the function $g(\cdot)$ determines the shape of the composite demand function $\hat{Q}(\cdot)$, while the functions $h(\cdot)$ and $q(\cdot)$ combine to determine the composite price function $\phi(\cdot)$.

Expression (18) implies that cross- or own-price demand effects are

$$
\frac{\partial x_{i}}{\partial p_{j}}=\hat{Q}(\phi) \phi_{i j}+\hat{Q}^{\prime}(\phi) \phi_{i} \phi_{j} .
$$

\footnotetext{
${ }^{11}$ There is a unique vector of relative quantities that solves (17) provided that $h$ and $q$ are quasi-concave, with one or both of them strictly so.
} 
(Here, subscripts to $\phi$ denote its partial derivatives.) This is akin to the Slutsky equation from classical demand theory. The first term in (19) represents the substitution effect while staying on the same composite quantity (or consumer surplus) contour, and the second term represents the impact of a price rise on the composite quantity demanded. This second term is negative, while the first term is negative if $j=i$ and could be positive or negative when $j \neq i$. For instance, if utility is homothetic, then $\phi(p)$ is positive and homogeneous degree 1, and expression (19) has the sign of $\left\{\left(\phi \phi_{i j} / \phi_{i} \phi_{j}\right)-\left[-\phi \hat{Q}^{\prime}(\phi) / \hat{Q}(\phi)\right]\right\}$. Here the first term is the elasticity of substitution of demand and the second term is the elasticity of composite demand, and the relative sizes of these two elasticities determine the sign of cross-price effects.

Since the quantity vector $x(p)$ induces inverse demand $p$, substituting (18) into the expression for inverse demand (12), and recalling that for positive demand we have $g^{\prime}(\hat{Q}(\phi)) \equiv \phi$, reveals that

$$
p \equiv \nabla h\left(x^{*}(p)\right)+\phi(p) \nabla q\left(x^{*}(p)\right) .
$$

This expression is also the first-order condition for problem (17). From (12), the prices that induce relative quantities $x^{*}$ lie on the straight line $\phi: \mapsto \nabla h\left(x^{*}\right)+\phi \nabla q\left(x^{*}\right)$, while $\phi$ determines how far along the line the price vector lies. Expression (20) is therefore the analogue for prices of the change of coordinates for quantities in (15) and decomposes the price vector $p$ into the composite price, $\phi(p)$, and "relative prices," which can be taken to be the straight line $\phi: \mapsto \nabla h\left(x^{*}\right)+\phi \nabla q\left(x^{*}\right)$ consisting of those prices that induce the same relative quantities $x^{*}$. In the particular case of homothetic utility, where $h \equiv 0$, these relative price lines are simply rays from the origin, just like relative quantities.

\section{B. Special Cases}

If $u(\cdot)$ is itself homothetic - for instance, if demand takes the familiar constant elasticity of substitution (CES) form - then (11) is trivially satisfied by setting $h \equiv 0$. In this case, expression (17) implies that $\phi(p)$ is homogeneous degree 1 , and $x^{*}(p)=\nabla \phi(p)$ is homogeneous degree 0 . More generally, homothetic demand is an instance of the subclass of (11) where $h$ takes the linear form $h(x)=a \cdot x$, when $\phi$ in (17) is homogeneous degree 1 in the "adjusted" price vector $(p-a)$.

Linear demand.-Another instance of this subclass with linear $h(\cdot)$ is linear demand, where utility $u(x)$ takes the quadratic form

$$
u(x)=a \cdot x-\frac{1}{2} x^{T} M x
$$

for constant vector $a>0$ and symmetric positive-definite matrix $M$. Here, inverse demands are $p(x)=a-M x$, and utility takes the form 
(11) by writing $h(x)=a \cdot x, q(x)=\sqrt{x^{T} M x}$, and $g(q)=-q^{2} / 2$. Here, $\nabla q(x)=M x / q(x)$, and so expression (12) implies that the set of price vectors that induce the same relative quantities takes the form $p=$ $a-t M x$ for scalar $t$, which are rays originating from the vector of choke prices $a$. It may be that $q(x)$ and therefore $s=q^{2} / 2$ decreases with $x_{i}$ when off-diagonal elements of $M$ are negative (which corresponds to products being complements).

Logit demand.-Suppose that consumer demand with prices $p$ takes the simple logit form

$$
x_{i}(p)=\frac{e^{\left(a_{i}-p_{i}\right) / \mu}}{k+\sum_{j} e^{\left(a_{j}-p_{j}\right) / \mu}},
$$

where $a=\left(a_{1}, \ldots, a_{n}\right)$ is a constant vector, $\mu>0$ is a parameter reflecting the substitutability of the products, and $k>0$ reflects the attractiveness of an outside option. ${ }^{12}$ Consumer surplus in terms of $p$ is therefore

$$
\mu \log \left(1+\frac{1}{k} \sum_{j} e^{\left(a_{j}-p_{j}\right) / \mu}\right) .
$$

If we write $q(x) \equiv \sum_{j} x_{j}$ for total quantity, adding (22) across products implies that the consumer surplus in (23) is given by

$$
s=-\mu \log (1-q(x)) .
$$

(As with any demand system resulting from discrete choice, the utility function is defined only on the domain $\Sigma_{i} x_{i} \leq 1$.) Therefore, consumer surplus is homothetic in quantity, and this demand system fits into our family.

Similar analysis applies with the more flexible nested logit specification (as studied in, e.g., Anderson and de Palma [1992]). To see this, suppose the products are partitioned into $G$ groups of products, so that consumer surplus in terms of prices takes the form

$$
\mu \log \left(1+\frac{1}{k} \sum_{g \in G} e^{v_{g} / \mu}\right),
$$

where

$$
v_{g}=\mu_{g} \log \left(\sum_{j \in g} e^{\left(a_{j}-p_{j}\right) / \mu_{g}}\right) .
$$

${ }^{12}$ Logit is also a leading example of the class of demand systems analyzed by Nocke and Schutz (2018) in which consumer surplus can be expressed as a sum of functions of individual prices. 
Here $\mu_{g}$ reflects substitution within the group $g$. As before, one can check that consumer surplus in (25) is given by (24), where $q(x)$ is the total quantity of all products.

With simple or nested logit, as with homogeneous goods, consumer surplus is a function only of total quantity $q(x)$. Since $\nabla q(x) \equiv(1, \ldots, 1)$, the set of prices that correspond to the same relative quantities takes the form $p+(t, \ldots, t)$, as can be seen directly from (22). This contrasts with the subclass with linear $h(x)$, where these lines were not parallel but emanated from a point.

Systems of complementary products.-A common situation is one in which consumers purchase a single unit of a "base product" and then combine this with variable quantities of one or more complementary products. For instance, consumers may need to gain entry to a theme park before they can go on the rides (Oi 1971) or need to buy a printer along with a suitable quantity of ink in order to print. To illustrate how these situations sometimes fit into our framework, suppose there is a continuum of consumers indexed by scalar $\theta$, where the type $\theta$ consumer has gross utility $U(y)+\theta$ if she consumes quantity $y$ of the combined service. (The following discussion also applies if $y$ is a vector of multiple services.) Adding over the population of consumers implies that aggregate gross utility if $x_{1}$ consumers (those with the highest value of $\theta$ ) each consume quantity $y$ of combined service takes the form $x_{1} U(y)+g\left(x_{1}\right)$ for some increasing concave function $g(\cdot)$, where $g$ is determined by the distribution of $\theta$. If $x_{2}$ denotes the quantity of combined service across all consumers, so that $x_{2}=x_{1} y$, it follows that aggregate utility in terms of the quantity vector $\left(x_{1}, x_{2}\right)$ is

$$
u\left(x_{1}, x_{2}\right)=x_{1} U\left(x_{2} / x_{1}\right)+g\left(x_{1}\right) .
$$

Clearly, this utility function fits into our family (11), where $h(x)=$ $x_{1} U\left(x_{2} / x_{1}\right)$ and composite quantity is just $q(x)=x_{1}$. This is another instance of the subclass with linear $q(x)$, but here $s(x)$ is a function only of $x_{1}$, the number of active consumers. The set of prices that correspond to the same relative quantities - that is, the same usage per active consumer-are horizontal lines with $p_{2}$ constant.

\section{Analysis}

In this section we discuss how to maximize welfare and monopoly profit, as well as calculate oligopoly outcomes, when the demand system satisfies (11) and the cost function satisfies

$$
c(x) \text { is convex and homogeneous degree } 1 .
$$


Consider again the problem of maximizing a weighted sum of profit and consumer surplus. If $0 \leq \alpha \leq 1$ is the relative weight on consumer surplus, the Ramsey objective (4) is

$$
\begin{aligned}
{[r(x)-c(x)]+\alpha s(x)=} & \alpha g(q(x))+(1-\alpha) g^{\prime}(q(x)) q(x) \\
& -q(x) \frac{c(x)-h(x)}{q(x)} .
\end{aligned}
$$

Here, we used the formulas (13) and (14).

As with the consumer's problem in Section III.A, the Ramsey problem can conveniently be solved by means of the change of variables (15). Expression (28) shows how the Ramsey objective can be written in terms of composite quantity $q(x)$ and the relative quantities $x / q(x)$. Expression (28) is decreasing in the term $[c(x)-h(x)] / q(x)$, and so relative quantities should be chosen to minimize this term. As in (17), write

$$
\kappa=\min _{x \geq 0}: \frac{c(x)-h(x)}{q(x)},
$$

which is solved by choosing relative quantities $x=x^{*}$, say, that satisfy the first-order condition ${ }^{13}$

$$
\kappa \nabla q\left(x^{*}\right)=\nabla c\left(x^{*}\right)-\nabla h\left(x^{*}\right)
$$

(Since the quantity vector that minimizes [29] and solves [30] is indeterminate up to a scaling factor, as in Sec. III.A, we normalize $x^{*}$ so that $q\left(x^{*}\right)=1$.) We deduce that maximizing any Ramsey objective involves choosing the same relative quantities $x^{*}$. In particular, profit-maximizing quantities are proportional to the efficient quantities corresponding to marginal-cost pricing. That is, the unregulated firm has an incentive to choose its relative quantities in an efficient manner, and the sole inefficiency arises from its supplying too little composite quantity.

Given this choice for relative quantities, the optimal choice for composite quantity $Q$ is easily derived. Expression (28) with relative quantities $x^{*}$ is the function

$$
\alpha g(Q)+(1-\alpha) g^{\prime}(Q) Q-\kappa Q
$$

(A sufficient condition for [31] to be concave in $Q$ for all $\alpha$ is that "composite revenue" $g^{\prime}(Q) Q$ be concave.) The vector of quantities that solves

\footnotetext{
${ }^{13}$ The minimization problem (29) might involve some products not being supplied, but without loss of generality we can leave such products out of the analysis. For if $u(x)$ satisfies (11), then the modified setting in which a subset of products is removed also satisfies (11).
} 
the Ramsey problem is then $Q x^{*}$, where $Q$ maximizes expression (31). ${ }^{14}$ The optimal composite quantity $Q$ increases with $\alpha$ and decreases with $\kappa$ and satisfies the Lerner formula

$$
\frac{g^{\prime}(Q)-\kappa}{g^{\prime}(Q)}=(1-\alpha) \eta(Q),
$$

where

$$
\eta(Q) \equiv-\frac{Q g^{\prime \prime}(Q)}{g^{\prime}(Q)}
$$

is the elasticity of inverse demand for composite quantity. Note that from (14) we have $\eta(Q) g^{\prime}(Q)=S^{\prime}(Q)$, where $S(Q)$ is consumer surplus as a function of composite quantity.

Since relative quantities are the same in all Ramsey problems, so too are relative price-cost margins. More precisely, the price-cost margins in the Ramsey problem with composite quantity $Q$ (which depends on $\alpha$ via expression [32]) are

$$
p\left(Q x^{*}\right)-\nabla c\left(Q x^{*}\right)=\left[g^{\prime}(Q)-\kappa\right] \nabla q\left(x^{*}\right) .
$$

This follows from expressions (12) and (30) and the fact that $\nabla c\left(Q x^{*}\right)=\nabla c\left(x^{*}\right)$. These margins are proportional to $\nabla q\left(x^{*}\right)$ and shrink equiproportionately when $\alpha$ and $Q$ are larger. Product $i$ is used as a loss leader, in the sense that its price is below marginal cost, in each Ramsey problem when composite quantity $q$ decreases with $x_{i}$ at $x^{*}$.

By virtue of the Ramsey-Cournot result in proposition 1, these properties extend to symmetric Cournot oligopoly. To summarize, we have the following proposition:

Proposition 3. Suppose that utility takes the form (11) and cost takes the form (27). As more weight is placed on consumer surplus in the Ramsey problem, each individual quantity increases equiproportionately and each price-cost margin contracts equiproportionately. The same is true in symmetric Cournot equilibrium as the number of firms increases.

An important special case involves constant marginal costs, so that $c(x) \equiv c \cdot x$ for a constant vector of marginal costs $c=\left(c_{1}, \ldots, c_{n}\right)$. In this case, $\kappa$ in (29) is simply $\phi(c)$, where the function $\phi(\cdot)$ is defined in (17), while $x^{*}=x^{*}(c)$. In this context, consider how optimal prices relate to

\footnotetext{
${ }^{14}$ The equiproportionate expansion of Ramsey quantities with $Q$ is reminiscent of, but distinct from, Gorman's (1961) linear income expansion paths (Engel curves) for quantities. Gorman's preference family was such that the consumer's expenditure function took the form $e(p, u)=a(p)+u b(p)$, where $a$ and $b$ are homogeneous degree 1 , while our family has gross utility of the form $u(x)=h(x)+g(q(x))$, where $h$ and $q$ are homogeneous degree 1.
} 
the firm's costs. This analysis is most transparent using the change of variables for prices (and costs) in (20), so that $\phi(p)$ is the composite price and the relative prices are the straight line that induces the same relative quantities $x^{*}$. Since in a Ramsey problem for our demand family it is optimal to choose relative quantities equal to those that correspond to efficient marginal-cost pricing, it is optimal to choose prices to lie on the same relative price line as the cost vector, so that $x^{*}(p)=x^{*}(c)$. The optimal markup of composite price over composite cost is then given by (32) after writing $\phi(p)=g^{\prime}(Q)$ and $\phi(c)=\kappa$. The optimal composite price, $\phi(p)$, decreases with the weight on consumer surplus, $\alpha$, and increases with composite cost, $\phi(c) .{ }^{15}$

What does this mean for the relationship between optimal prices and costs? Expressions (32) and (34) imply that optimal prices satisfy

$$
p-c=(1-\alpha) \eta(Q) g^{\prime}(Q) \times \nabla q\left(x^{*}(c)\right) .
$$

Consider first the subclass in which $h$ takes the linear form $h(x)=a \cdot x$. Since optimal quantities are $Q x^{*}(c)$, expression (12) shows that prices satisfy $p-a=g^{\prime}(Q) \nabla q\left(x^{*}(c)\right)$, which together with (35) implies that

$$
p-c=\frac{(1-\alpha) \eta(Q)}{1-(1-\alpha) \eta(Q)} \times(c-a) .
$$

In particular, when preferences are homothetic, so that $a=0$, we obtain the familiar result that proportional price-cost markups are the same for each product.

In the isoelastic case in which $\eta$ is constant, expression (36) implies that the optimal price for product $i$ depends only on $c_{i}$, not on any other product's cost, and so there is no cross-cost pass-through in prices, even though there may be substantial cross-price effects in the demand system. For instance, if $u$ is homothetic and $g(Q)=(1 / \gamma) Q^{\gamma}$, where $0<\gamma<$ 1 , then $\eta \equiv 1-\gamma$ and (36) implies that the most profitable prices (i.e., when $\alpha=0$ ) are

$$
p=\frac{1}{\gamma} c
$$

Likewise, with linear demand we have $\eta \equiv-1$, and expression (36) implies that the profit-maximizing prices are

\footnotetext{
${ }^{15}$ Since the profit-maximizing firm's choice of composite quantity falls with $\phi(c)$ and since consumer surplus, $s$, is an increasing function of composite quantity, we deduce that the firm necessarily offers a lower level of consumer surplus when unit cost $c_{i}$ increases. Our family of demand systems therefore excludes the possibility explored by Edgeworth (1925) that imposing a linear tax on a product supplied by a multiproduct monopolist might induce the firm to lower all of its prices.
} 


$$
p=\frac{1}{2}(a+c)
$$

As well as cross-cost effects being absent, these prices do not depend on the matrix $M$ of own- and cross-price parameters. Provided that the seller can profitably supply product $i$ on its own, in these cases the firm's most profitable price for product $i$ when it supplies all products is the same as in the situation in which it can supply only this product. ${ }^{16}$ More generally within this subclass with linear $h$, expression (36) and the fact that the most profitable $Q$ decreases with each cost imply that all cross-cost pass-through terms for $p_{i}$ have the same sign as $\left(a_{i}-c_{i}\right) \eta^{\prime}(Q)$.

Alternatively, consider the subclass in which composite quantity takes the linear form $q(x)=b \cdot x$. Then (35) implies that

$$
p-c=(1-\alpha) S^{\prime}(Q) \times b \text {. }
$$

In the example with complementary products in which utility is (26) and $b=(1,0)$, this expression implies there is marginal-cost pricing for usage $\left(p_{2}=c_{2}\right)$, and in particular, changes in $c_{1}$ have no impact on $p_{2}$. However, there is cross-cost pass-through in the other direction: since the optimal $Q$ decreases with both costs, it follows that $p_{1}$ increases or decreases with $c_{2}$ according to whether $S(Q)$ is concave or convex. In the simple or nested logit example (see $[24]$ ) we have $b=(1, \ldots, 1)$, so the price-cost margin $p_{i}-c_{i}$ is the same for each product $i$, and the common markup $(1-\alpha) S^{\prime}(Q)=\mu(1-\alpha) /(1-Q)$ increases with $Q$. Since the optimal $Q$ decreases with each $c_{i}$, it follows that cross-cost pass-through with logit demand is negative and the most profitable price for one product decreases with the cost of each other product.

As shown in proposition 1, with $m$ firms each with a cost function satisfying (27), the outcome with Cournot oligopoly coincides with the Ramsey optimum if the weight on consumer interests in the Ramsey problem is set equal to $\alpha=(m-1) / m$. When $c(x)=c \cdot x$ and $h(x)=$ $a \cdot x$, expression (36) implies that

$$
p-c=\frac{\eta(Q)}{m-\eta(Q)} \times(c-a) .
$$

${ }^{16}$ The proviso about the profitability of single-product supply is unnecessary when all products are substitutes. But when products are complements, the removal of product $j$ could cause product $i$ to become unprofitable on a standalone basis. Consider, for instance, the symmetric demand system with two products given by $p_{i}=\frac{2}{3}\left(1-2 x_{i}+x_{j}\right)$. With the cost vector $c=\left(\frac{5}{6}, 0\right)$ the most profitable prices are $p=\left(\frac{3}{4}, \frac{1}{3}\right)$ and there is a subsidy offered for the first product. Clearly, if the multiproduct firm chooses a price below cost for some product, in the situation in which it supplies only this product, it could not profitably do so. See Amir et al. (2016) for a detailed investigation of multiproduct monopoly with linear demand. 
As in the Ramsey problem, cross-cost pass-through terms for product $i$ have the same sign as $\left(a_{i}-c_{i}\right) \eta^{\prime}(Q)$. (Here, a change in one product's cost is assumed to be industrywide, not firm specific.) So if $\eta(Q)$ is constant, then the equilibrium price for one product does not depend on the costs of other products. ${ }^{17}$

Firm-specific cost shocks can be analyzed using expression (10). Thus, provided that each firm supplies all products in equilibrium, when $h(x)=a \cdot x$, expression (39) continues to apply provided that the vector $c$ is interpreted to be the industry average vector of marginal costs. To illustrate, with linear demand the equilibrium price for product $i$ with $m$ firms is

$$
p_{i}=\frac{1}{m+1}\left(a_{i}+\sum_{j} c_{i}^{j}\right),
$$

where $c_{i}^{j}$ is firm $j$ 's cost for product $i$. Thus an increase in cost $c_{i}^{j}$ will be passed through at rate $1 /(m+1)$ to product $i$ 's price and will have no impact on prices for other products.

\section{Regulation with Asymmetric Cost Information}

When the demand system belongs to the family (11), we have seen that an unregulated monopolist will choose an efficient pattern of relative quantities, even though it supplies too little composite quantity. This suggests that, in some circumstances at least, the optimal way to regulate market power when the firm has private information is to control only its composite quantity, leaving it free to choose relative quantities to reflect its private information.

To explore this issue, we consider optimal regulation of multiproduct monopoly by extending the analysis of the single-product case by Baron and Myerson (1982). Specifically, there is common knowledge about the demand system, but the firm has private information about its cost function. In particular, suppose the firm has the vector of constant marginal costs, $c=\left(c_{1}, \ldots, c_{n}\right)$, which the firm knows but which is not observed by the regulator. Optimal regulation can be analyzed by way of a "direct" scheme whereby the firm reports its cost vector to the regulator, and conditional on this report, the firm is instructed to supply a vector of quantities $X$ and receives a transfer $T$ funded by consumers. The revelation principle implies that we can restrict attention to mechanisms in which the firm is given an incentive to report its cost vector truthfully. The reg-

\footnotetext{
${ }^{17}$ In contrast to the macroeconomics literature on price stickiness arising, e.g., from menu costs, the lack of (cross-) cost pass-through here is in a setting with freely flexible prices.
} 
ulator places weight $0 \leq \beta \leq 1$ on profit relative to consumer surplus, where profit includes the transfer $T$ and consumer surplus includes the deduction for the transfer $T$.

For simplicity, suppose that preferences are homothetic, so that $h \equiv 0 .{ }^{18}$ In this case, the cost vector can be written in the "polar coordinates" form

$$
c=\phi(c) \times \frac{c}{\phi(c)},
$$

where $\phi(c)$ is the composite cost function, which is homogeneous degree 1 . Here, the vector $c / \phi(c)$ represents the firm's "relative costs," that is, the ray from the origin on which $c$ lies. As discussed earlier in an unregulated context, the coordinate $\phi(c)$ determines the firm's incentive to set its composite price while its choice of relative prices is equal to $c / \phi(c)$.

It is useful to consider first the situation in which the firm's relative cost coordinate in (40) is directly observed by the regulator, before turning to the more realistic situation in which it is not. ${ }^{19}$ Therefore, suppose that the regulator knows that the firm's cost vector lies on the ray $c / \phi(c)$, but not how far along this ray the cost vector lies. The regulator's problem is then a one-dimensional screening problem, which can be solved using by-now standard arguments in Baron and Myerson (1982, sec. 3). ${ }^{20}$ Specifically, suppose the regulator knows that the firm's cost vector lies on the ray corresponding to relative costs $c / \phi(c)$ and believes the firm's composite cost $\kappa=\phi(c)$ has cumulative distribution function $F(\kappa \mid c / \phi(c))$ and associated density function $f(\kappa \mid c / \phi(c))$. Provided that (41) weakly increases with $\kappa$, optimal regulation instructs the firm with composite cost $\kappa$ to charge the composite price

$$
\phi(p)=\kappa+(1-\beta) \frac{F(\kappa \mid c / \phi(c))}{f(\kappa \mid c / \phi(c))}
$$

and leaves the firm free to choose its relative prices, which the firm therefore chooses to be the efficient relative prices lying on the same ray $c / \phi(c)$.

${ }_{18}$ An argument similar to that used in the following applies also when $h \neq 0$ but is somewhat less transparent.

${ }_{19}$ This approach is similar to that in Armstrong (1996, sec. 4.4) and Armstrong and Vickers (2001, proposition 5), where a multidimensional screening problem is solved by supposing that the principal can observe all but one dimension of the agent's private information, and then finding conditions that ensure that the incentive scheme offered to these agents does not actually depend on the observed parameters.

${ }^{20}$ Further details can be found in our working paper (Armstrong and Vickers 2016). Baron and Myerson (1982) also show how to solve the problem when (41) is not increasing. Sappington (1983) solves a distinct multiproduct regulation problem with scalar private information, where the firm can affect the balance between fixed and variable costs. 
Consider now the case in which the regulator cannot observe the direction coordinate $c / \phi(c)$. In general, with current techniques this seems to be an intractable problem. However, in the special case in which the distribution for $c$ is such that the two coordinates in (40) are stochastically independent, the optimal composite price in (41) does not depend on the ray $c / \phi(c)$. Therefore, the above regulatory scheme continues to be optimal even when relative costs are private information. We therefore make the following deduction:

Proposition 4. Suppose the distribution for $c$ is such that $\phi(c)$ and $c / \phi(c)$ are stochastically independent, where the firm's composite cost $\kappa=\phi(c)$ has cumulative distribution function $F(\kappa)$ and associated density function $f(\kappa)$. Provided that (42) weakly increases with $\kappa$, optimal regulation requires the firm with composite cost $\kappa$ to charge the composite price

$$
\phi(p)=\kappa+(1-\beta) \frac{F(\kappa)}{f(\kappa)}
$$

and to be free to set its relative prices.

This optimal scheme controls the firm's composite price but does not attempt to influence its choice of relative prices. Intuitively, when $\phi(c)$ and $c / \phi(c)$ are stochastically independent, the firm is happy for the regulator to observe its relative costs but not its composite cost $\phi(c)$. That is, for a given composite price, the firm and the regulator have aligned preferences with respect to the choice of relative prices. The regulator gains by delegating to the firm the choice of relative prices, to enable the firm to make use of its private information about relative costs. However, if $\phi(c)$ and $c / \phi(c)$ were correlated, observing the firm's relative costs is informative about the firm's composite cost, which gives the firm an incentive to misreport its relative costs as well as its composite cost.

To illustrate proposition 4, suppose that there are two products and utility takes the additive form $u(x)=\sqrt{x_{1}}+\sqrt{x_{2}}$, which is homothetic with composite quantity $q(x)=\left(\sqrt{x_{1}}+\sqrt{x_{2}}\right)^{2}$ and so

$$
\kappa=\phi(c)=\frac{1}{\left(1 / c_{1}\right)+\left(1 / c_{2}\right)} .
$$

The method requires that the distribution for $\left(c_{1}, c_{2}\right)$ be such that $\left(1 / c_{1}\right)+\left(1 / c_{2}\right)$ and $c_{1} / c_{2}$ are independent variables. Since the sum and ratio of two independent and identically distributed exponential variables are independent, the method works if each $c_{i}$ is an independent draw from a distribution such that $1 / c$ is exponentially distributed. ${ }^{21}$ Specifically, suppose that each $c_{i}$ has support $[0, \infty)$ and cumulative distribu-

${ }^{21}$ That is, $c_{i}$ comes from an inverse- $\chi^{2}$ distribution with two degrees of freedom. 
tion function $(\mathrm{CDF}) \operatorname{Pr}\{c \leq t\}=e^{-1 / t}$. Then $1 / \kappa$ is the sum of two exponential variables, and so $\kappa$ has $\operatorname{CDF} F(\kappa)=[1+(1 / \kappa)] e^{-1 / \kappa}$ and corresponding density $f(\kappa)=\left(1 / \kappa^{3}\right) e^{-1 / \kappa}$. Expression (42) then implies that the optimal composite price for the type $\kappa$ firm is $\kappa+(1-\beta) \kappa^{2}(1+\kappa)$, which increases with $\kappa$ as required, which implies that the optimal individual prices are

$$
p_{i}=c_{i}[1+(1-\beta) \kappa(1+\kappa)],
$$

where $\kappa$ is the function of $c$ in (43). ${ }^{22}$

In expression (44) there is marginal-cost pricing for those firms with $\kappa=0$, and so $p_{i}=c_{i}$ whenever the other product has $c_{j}=0$. The regulated price for one product is an increasing function of the other product's cost, even though this demand system has no cross-price effects and there is no statistical correlation in costs across products. Note also that all types of firms participate in the mechanism, and in contrast to Armstrong (1996, sec. 3), there is no "exclusion" in the optimal scheme. However, as long as $\beta<1$, when the firm has sufficiently high costs, the prices in (44) are above the unregulated profit-maximizing prices (which with this demand system are $p_{i}=2 c_{i}$ ), a possibility that was noted in the single-product context by Baron and Myerson $(1982,292)$.

\section{Conclusions}

In this paper we have studied a range of multiproduct pricing questions in terms of consumer surplus considered as a function of quantities. With a monopolist, this is the classical profit-maximization problem, or more generally the Ramsey problem of welfare maximization subject to a profit constraint. With oligopoly, by contrast, the multiproduct pricing question is one of equilibrium among independent firms, not optimization by a single decision maker. We have shown, however, that in perhaps the most natural multiproduct oligopoly model, with symmetric Cournot firms, there is Ramsey-Cournot equivalence. With a suitable weighting between profit and consumer surplus, Ramsey quantities are Cournot equilibrium quantities, and vice versa. Solutions to Ramsey optimization problems are therefore Cournot equilibrium solutions too.

The other main aim of the paper has been to show how multiproduct monopoly analysis is made simpler when consumer surplus is a homothetic function of quantities. Whether the firm's objective is profit or a Ramsey combination of profit and consumer surplus, it optimizes by se-

\footnotetext{
22 Another example that works nicely is $q(x)=\sqrt{x_{1} x_{2}}$, so that $\phi(c)=2 \sqrt{c_{1} c_{2}}$. In this case proposition 4 applies when the distribution for $\left(c_{1}, c_{2}\right)$ is such that the product $c_{1} c_{2}$ and the ratio $c_{1} / c_{2}$ are stochastically independent, which is the case when each $c_{i}$ is an independent draw from a lognormal distribution.
} 
lecting efficient (i.e., welfare-maximizing) quantities scaled back equiproportionately. The resulting optimal markups yield, for example, transparent results on multiproduct cost pass-through, including instances without cross-cost pass-through. With Ramsey-Cournot equivalence, these results extend to the Cournot oligopoly setting with symmetric firms. The family of demand systems also provides a natural basis on which to explore the intuition that regulation of multiproduct monopoly should focus on the general level of prices and not the pattern of relative prices, and we showed when that intuition is precisely correct.

The family of demand systems with consumer surplus a homothetic function of quantities is of course restrictive. But it includes a number of familiar yet diverse special cases, including CES, linear demand, and logit. Moreover, it shows how those special cases are themselves instances of wider subclasses of demand systems, involving $h(x) \equiv 0$, linear $h(x)$, and linear $q(x)$, respectively. Analysis using the demand family could be extended to various settings beyond the scope of this paper. For example, questions about product range could be explored by introducing fixed costs per product, and the framework could be a basis for the study of the welfare effects of price discrimination in multiproduct settings.

\section{Appendix}

\section{Proof of Proposition 1}

We first rule out asymmetric equilibria. Consider a candidate asymmetric equilibrium in which $x$ is the total supply and in which two firms, $j$ and $k$, supply distinct quantity vectors $x^{j}$ and $x^{k}$. At equilibrium it cannot be profitable for $j$ to deviate to supplying $y^{j}(\varepsilon) \equiv x^{j}+\varepsilon\left(x^{k}-x^{j}\right)$, where $\varepsilon$ is a scalar. Thus $\varepsilon=0$ must maximize j's profit

$$
y^{j}(\varepsilon) \cdot p\left(x+\varepsilon\left(x^{k}-x^{j}\right)\right)-c\left(y^{j}(\varepsilon)\right),
$$

which has first-order condition

$$
\left[p(x)+x^{j} \cdot D p(x)-\nabla c\left(x^{j}\right)\right] \cdot\left(x^{k}-x^{j}\right)=0 .
$$

Adding the corresponding condition for firm $k$ yields the contradiction

$$
0=-\left(x^{k}-x^{j}\right) \cdot D p(x) \cdot\left(x^{k}-x^{j}\right)+\left[\nabla c\left(x^{k}\right)-\nabla c\left(x^{j}\right)\right] \cdot\left(x^{k}-x^{j}\right)>0,
$$

where the inequality follows from the negative-definiteness of the matrix $D p(x)$ together with the convexity of $c(x)$. We deduce that $x$ cannot be equilibrium supply unless it is symmetrically shared between firms.

Turning to equilibrium existence, note that the Ramsey objective (7) can be written $(1-\alpha) r(x)+\alpha u(x)-m c((1 / m) x)$. Suppose that quantity vector $x$ solves this Ramsey problem when $\alpha=(m-1) / m$. Since $c$ is convex, it follows that choosing $x^{j}=(1 / m) x$ for each $j$ maximizes 


$$
\frac{1}{m} r\left(\sum_{j} x^{j}\right)+\frac{m-1}{m} u\left(\sum_{j} x^{j}\right)-\sum_{j} c\left(x^{j}\right) .
$$

In particular, choosing $y=x$ maximizes the function

$$
\rho(y, x) \equiv \frac{1}{m} r\left(x+\frac{1}{m}(y-x)\right)+\frac{m-1}{m} u\left(x+\frac{1}{m}(y-x)\right)-c\left(\frac{1}{m} y\right) .
$$

From the concavity of $u$ we have for all $y$ and $z$ that

$$
\begin{aligned}
\rho(y, z)-\frac{m-1}{m} u(z) & =\frac{1}{m} r\left(z+\frac{1}{m}(y-z)\right)+\frac{m-1}{m}\left[u\left(z+\frac{1}{m}(y-z)\right)-u(z)\right]-c\left(\frac{1}{m} y\right) \\
& \geq \frac{1}{m}\left[z+\frac{1}{m}(y-z)+\frac{m-1}{m}(y-z)\right] \cdot p\left(z+\frac{1}{m}(y-z)\right)-c\left(\frac{1}{m} y\right) \\
& =\frac{1}{m} y \cdot p\left(z+\frac{1}{m}(y-z)\right)-c\left(\frac{1}{m} y\right) \\
& \equiv \pi(y, z),
\end{aligned}
$$

where $\pi(y, z)$ is the profit of a Cournot firm that supplies quantity vector $(1 / m) y$ when its rivals each supply $(1 / m) z$. Since

$$
\rho(z, z)-\frac{m-1}{m} u(z)=\pi(z, z),
$$

we have that

$$
\pi(z, z)-\pi(y, z) \geq \rho(z, z)-\rho(y, z) .
$$

Thus the Cournot firm has a stronger desire than the Ramsey objective to be symmetric with other firms. With $z=x$, the Ramsey optimal quantities, (A1) yields

$$
\pi(x, x)-\pi(y, x) \geq \rho(x, x)-\rho(y, x) \geq 0,
$$

where the final inequality follows from $x$ being Ramsey optimal. We deduce that it is a Cournot equilibrium for each firm to supply $(1 / m) x$.

Finally, consider the uniqueness of equilibrium. We have already shown there are no asymmetric equilibria, while expression (9) shows that any symmetric equilibrium satisfies the first-order conditions for maximizing the Ramsey objective (7) with $\alpha=(m-1) / m$. This Ramsey objective can be written as

$$
\frac{1}{m} r(x)+\frac{m-1}{m} u(x)-m c\left(\frac{1}{m} x\right),
$$

and given that $u$ is strictly concave and $c$ is convex, this is strictly concave if $r(x)$ is concave. In this case, there is a unique quantity vector $x$ that satisfies the firstorder condition (9) and, hence, a unique symmetric equilibrium.

\section{Proof of Proposition 2}

To show necessity of (11), suppose that consumer surplus $s(x)$ is homothetic, so that $s(x) \equiv G(q(x))$ for some increasing function $G$ and some function $q(x)$ that is homogeneous degree 1 . We can write $G$ as $G(q) \equiv g(q)-q g^{\prime}(q)$ for an- 
other function $g(\cdot)$. Given any function $G(\cdot)$, one can generate the corresponding $g(\cdot)$ using the procedure

$$
g(q)=-q \int^{q}\left[G(\tilde{q}) / \tilde{q}^{2}\right] d \tilde{q} .
$$

This function $g(q)$ is concave given that $G(q)$ is increasing. Then

$$
s(\tilde{x} / k)=g(q(\tilde{x}) / k)-\frac{q(\tilde{x})}{k} g^{\prime}(q(\tilde{x}) / k)=\frac{d}{d k} \operatorname{kg}(q(\tilde{x}) / k) .
$$

Note that (1) can be generalized slightly so that $s(\tilde{x} / k)=(d / d k) k u(\tilde{x} / k)$, and so (A2) can be integrated to yield

$$
k u(\tilde{x} / k)=h(\tilde{x})+k g(q(\tilde{x}) / k)
$$

for some constant of integration $h(\tilde{x})$. Writing $x=\tilde{x} / k$, this becomes

$$
u(x)=\frac{h(k x)}{k}+g(q(x)) .
$$

Since this holds for all $k$, we deduce that $u(x)=h(x)+g(q(x))$, where $h(x)$ is homogeneous degree 1.

\section{References}

Amir, Rabah, Jim Jin, Gerald Pech, and Michael Tröge. 2016. "Prices and Deadweight Loss in Multiproduct Monopoly." L. Public Econ. Theory 18:364-62.

Anderson, Simon, and André de Palma. 1992. "Multiproduct Firms: A Nested Logit Approach.” I. Indus. Econ. 40:261-76.

Armstrong, Mark. 1996. "Multiproduct Nonlinear Pricing." Econometrica 64:5175 .

Armstrong, Mark, and John Vickers. 2001. "Competitive Price Discrimination." Rand I. Econ. 32:579-605.

. 2016. "Multiproduct Pricing Made Simple.” Discussion Paper no. 68171, Munich Personal RePEc Archive.

Baron, David, and Roger Myerson. 1982. "Regulating a Monopolist with Unknown Costs." Econometrica 50:911-30.

Baumol, William, and David Bradford. 1970. "Optimal Departures from Marginal Cost Pricing." A.E.R. 60:265-83.

Bergstrom, Theodore, and Hal Varian. 1985. "Two Remarks on Cournot Equilibria." Econ. Letters 19:5-8.

Besanko, David, Jean-Pierre Dubé, and Sachin Gupta. 2005. "Own-Brand and Cross-Brand Retail Pass-Through.” Marketing Sci. 24:123-37.

Bliss, Christopher. 1988. "A Theory of Retail Pricing." I. Indus. Econ. 36:375-91.

Bulow, Jeremy, and Paul Klemperer. 2012. "Regulated Prices, Rent Seeking, and Consumer Surplus.” L.P.E. 120:160-86.

De Fraja, Gianni. 1996. "Product Line Competition in Vertically Differentiated Markets.” Internat. I. Indus. Org. 14:389-414.

Edgeworth, Francis. 1925. Papers Relating to Political Economy. London: Macmillan. Gorman, W. M. 1961. "On a Class of Preference Fields.” Metroeconomica 13:53-56. Johnson, Justin, and David Myatt. 2006. "Multiproduct Cournot Oligopoly." Rand I. Econ. 37:583-601. 
Laffont, Jean-Jacques, and Jean Tirole. 1993. A Theory of Incentives in Procurement and Regulation. Cambridge, MA: MIT Press.

Mirrlees, James. 1976. "Optimal Tax Theory: A Synthesis.” L. Public Econ. 6:32758.

Monderer, Dov, and Lloyd Shapley. 1996. "Potential Games." Games and Econ. Behavior 14:124-43.

Moorthy, Sridhar. 2005. "A General Theory of Pass-Through in Channels with Category Management and Retail Competition.” Marketing Sci. 24:110-22.

Morris, Stephen, and Takashi Ui. 2004. "Best Response Equivalence." Games and Econ. Behavior 49:260-87.

Nocke, Volker, and Nicolas Schutz. 2018. "Multiproduct-Firm Oligopoly: An Aggregative Games Approach.” Econometrica 86:523-57.

Oi, Walter. 1971. "A Disneyland Dilemma: Two-Part Tariffs for a Mickey Mouse Monopoly." O. J.E. 85:77-96.

Ramsey, Frank. 1927. "A Contribution to the Theory of Taxation." Econ.J. 37:4761.

Sappington, David. 1983. "Optimal Regulation of a Multiproduct Monopoly with Unknown Technological Capabilities.” Bell I. Econ. 14:453-63.

Slade, Margaret. 1994. "What Does an Oligopoly Maximize?" I. Indus. Econ. 42:45-61.

Vives, Xavier. 1999. Oligopoly Pricing. Cambridge, MA: MIT Press.

Weyl, Glen, and Michal Fabinger. 2013. "Pass-Through as an Economic Tool: Principles of Incidence under Imperfect Competition.” L.P.E. 121:528-83. 